Journal of Universal Mathematics

VOL.4 No.1 PP.81-86 (2021)

ISSN-2618-5660

DOI: $10.33773 /$ jum. 746512

\title{
FUZZY LINEAR MAPPINGS
}

\author{
ÜMİT DENIZ
}

\begin{abstract}
In this paper we study fuzzy linear mappings. In literature the fuzzy linear mapping definition was given with classic functions. But we give the definition of fuzzy linear mapping with using the definition of fuzzy function that Demirci [4] was given before. With this definition, we give some definition and theorems that similar to classic algebra about linear mappings and prove them.
\end{abstract}

\section{INTRODUCTION}

The concept of a fuzzy set was introduced by Zadeh [8]. Fuzzy sets gives opportunity to constitute the uncertain problems in real life to mathematical models. Most of the problems in engineering, economics, medical science etc, have various uncertainties. The fuzzy set theory helps to modelling and solving these problems. Many mathematician tried to transfer the classic set theory to use the definition of Zadeh' s fuzzy set. Rosenfeld [15] gave the definition of fuzzy groups and fuzzy grupoids. Liu [16], [17] gave the definition of fuzzy subrings and fuzzy ideals of a ring. Fuzzy relations are playing an important role in fuzzy modelling, fuzzy control and significant applications in relational databases, approximate reasoning, medical diagnosis. Several other authors have applied fuzzy sets to various branches of Mathematics. In this paper we apply Fuzzy linear mappings. Some mathematicians give the definition of fuzzy linear mapping definition with using classic functions as in [9]. In our definition we use the fuzzy function definition of Demirci [5].

\section{Preliminaries}

In this section we recall some definitions and results which will be used later. In first the fuzzy set definition is defined the researchers used infimum operation for their definitions and theorems. By time the researchers used t-norms instead of infimum operation. Here are some definitions and theorems of triangular norms (t-norm) explanined.

Definition 2.1. [10] Let $(L, \leq)$ be a complete lattice with top and bottom elements 1,0 , respectively. A triangular norm (briefly t-norm) is a binary operation $\mathrm{T}$ on $\mathrm{L}$ which is commutative, associative, monotone and has 1 as neutral element, i.e., it

Date: June, 2020.

Key words and phrases. Fuzzy Functions, Fuzzy Subspaces, Fuzzy Linear Mappings. 
is a function

$T: L \times L \rightarrow L$ such that for all $x, y, z \in L$,

(i) existence of neutral element 1: $T(x, 1)=x$;

(ii) monotonicity: $x \leq y \Rightarrow T(x, z) \leq T(y, z)$;

(iii) commutativity; $T(x, y)=T(y, x)$;

(iv) associativity: $T(x, T(y, z))=T(T(x, y), z)$.

Conditions (i) and (ii) imply that for any t-norm $T(x, y) \leq x$ and $T(x, y) \leq y$ hold, thus $T(x, y) \leq x \wedge y$, for all $x, y \in L$.

Example 2.2. (1) Standard intersection T-norm $T_{m}(x, y)=\min \{x, y\}$.

(2) Bounded sum T-norm $T_{b}(x, y)=\max \{0, x+y-1\}$.

(3) Algebraic product T-norm $T_{p}(x, y)=x y$.

(4) Drastic T-norm

$$
T_{D}(x, y)= \begin{cases}y & \text { if } x=1 \\ x & \text { if } y=1 \\ 0 & \text { otherwise }\end{cases}
$$

(5) Nilpotent minimum T-norm

$$
T_{n M}(x, y)= \begin{cases}\min \{x, y\} & \text { if } x+y>1 \\ 0 & \text { otherwise }\end{cases}
$$

(6) Hamacher product T-norm

$$
T_{H_{o}}(x, y)= \begin{cases}0 & \text { if } x=y=0, \\ \frac{x y}{x+y-x y} & \text { otherwise } .\end{cases}
$$

The drastic t-norm is the pointwise smallest t-norm and the minimum is the pointwise largest t-norm: $T_{D}(x, y) \leq T(x, y) \leq T_{\min }(x, y)$ for all $x, y \in[0,1]$.

Theorem 2.3. [6] Let $T$ be a t-norm. Then

$$
T(T(x, y), T(w, z)=T(T(x, w), T(y, z),
$$

for all $x, y, w, z \in L$.

Definition 2.4. [6]

(i) A t-norm $\mathrm{T}$ on a lattice $\mathrm{L}$ is called $\mathrm{V}$-distributive if $T(x, y \vee z)=T(x, y) \vee T(x, z)$ for all $x, y, z \in L$.

(ii) A t-norm $\mathrm{T}$ on a complete lattice $\mathrm{L}$ is called infinetly $\mathrm{V}$-distributive if $T\left(a, \underset{Q}{\vee} b_{\tau}\right)=\underset{Q}{\vee} T\left(a, b_{\tau}\right)$ for any subset $a, b_{\tau} \in L, \tau \in Q$ of $\mathrm{L}$.

Theorem 2.5. [7] Let $L$ be a triangular norm on lattice $L$ then

(i) $T(x, 0)=T(0, x)=0$ for all $x \in L$,

(ii) if $a \leq b$ and $x \leq y$ then $T(a, x) \leq T(b, y)$,

(iii) if $\left\{\bar{a}_{i} \mid i \in I\right\},\left\{\bar{b}_{i} \mid i \in I\right\} \subset L$ then $T\left(\bigwedge_{i \in I} a_{i}, \bigwedge_{i \in I} b_{i}\right) \leq \bigwedge_{i \in I} T\left(a_{i}, b_{i}\right)$.

Theorem 2.6. [8] Let $L$ be a complete lattice. If $T$ is an infinetly $\vee$-distributive t-norm then

$$
\bigvee_{i \in I j \in J} T\left(a_{i}, b_{j}\right)=T\left(\bigvee_{i \in I} a_{i}, \bigvee_{j \in J} b_{j}\right)
$$

Definition 2.7. [8] Let $\mathrm{L}$ be a complete lattice. By a L-subset of $\mathrm{X}$ we mean a function from $\mathrm{X}$ into $\mathrm{L}$. We denote all $\mathrm{L}$-subsets set by $F(X, L)$. In particular, when $\mathrm{L}$ is $[0,1]$, the $\mathrm{L}$-subsets of $\mathrm{X}$ are called fuzzy subsets. 
Definition 2.8. [7] If $\mathrm{X}$ and $\mathrm{Y}$ are sets then the function $f: X \times Y \rightarrow L$ is called a L-relation and the set of all L-relations is denoted by $F(X \times Y, L)$.

Definition 2.9. [4] Let $\mathrm{L}$ be a complete lattice. $E: X \times X \rightarrow L$ a L-relation $\mathrm{E}$ on a set $\mathrm{X}$ is a TL-equivalence relation if and only if for all a,b,c of $\mathrm{X}$ the following properties are satisfied:

$(E 1) E(a, a)=1$,

$(E 2) E(a, b)=E(b, a)$,

$(E 3) T(E(a, b), E(b, c)) \leq E(a, c)$.

$\mathrm{E}$ is called a seperable TL-equivalence relation or a TL-equality if, in addition, $(E 4) E(a, b)=1$ implies $a=b$. If $\mathrm{E}$ is a TL-equivalence relation on $\mathrm{X}$ it is shown by $(X, E)$.

Theorem 2.10. [4] Let $(X, E)$ and $(Y, F)$ be two equivalence relations. Then $T L$ subset $E \times F: X \times Y \rightarrow L$ defined by

$(E \times F)\left((x, y),\left(x^{\prime}, y^{\prime}\right)\right)=T\left(E\left(x, x^{\prime}\right), F\left(y, y^{\prime}\right)\right) \quad E \times F$ is a TL-equivalence relation.

Definition 2.11. [4] Let $\mathrm{E}$ be a TL-equivalence relation on a set X. A L-subset $\mu$ of $\mathrm{X}$ is extensional or observable w.r.t. E if and only if $T(\mu(y), E(x, y)) \leq \mu(x) \forall x, y \in$ $X$.

Definition 2.12. [7] Let $\mathrm{X}, \mathrm{Y}$ and $\mathrm{Z}$ be sets and $f: X \times Y \rightarrow L$ and $g: Y \times Z \rightarrow L$ be L-relations. Then $g \circ_{T} f: X \times Z \rightarrow L$ L-relation is called composition of $\mathrm{f}$ and g such that

$(g \circ T f)(x, z)=\bigvee_{y \in Y} T(f(x, y), g(y, z))$.

Definition 2.13. [5] Let $f: X \times Y \rightarrow L$ be a L-relation then we call the function $f^{-1}: Y \times Y \rightarrow L$ defined by $f^{-1}(y, x)=f(x, y)$ the inverse of $\mathrm{f}$ L-relation.

Definition 2.14. [5] Let $f: X \times Y \rightarrow L$ be a L-relation and $A \in F(X, L)$ and $B \in F(Y, L)$. The L-subsets $f(A)$ and $f^{-1}(B)$ defined by $\forall x \in X, y \in Y$

$f(A)(y)=\bigvee_{x \in X} T(A(x), f(x, y))$ and $f^{-1}(B)(x)=\bigvee_{y \in Y} T(B(y), f(x, y))$ are respectively the image of $\mathrm{A}$ and the inverse image of $\mathrm{B}$.

Definition 2.15. [5] Let $(X, E)$ and $(Y, F)$ be two TL-equivalence relations and $f \in F(X \times Y, L)$. Then

a) $\mathrm{f}$ is called E-extensional iff the inequality $T\left(f(x, y), E\left(x, x^{\prime}\right)\right) \leq f\left(x^{\prime}, y\right)$

forall $x, x^{\prime} \in X, y \in Y$ is satisfied. We denote all E-extensional L-relations set by $F(X \times Y, E, L)$.

b) $\mathrm{F}$ is called F-extensional iff the inequality $T\left(f(x, y), F\left(y, y^{\prime}\right)\right) \leq f\left(x, y^{\prime}\right) \quad \forall x \in$ $X, y, y^{\prime} \in Y$. We denote all F-extensional L-relations set by $F(X \times Y, F, L)$.

c) We call a $\mathrm{f}$ L-relation E-F-extensional if $\mathrm{f}$ is E-extensional and F-extensional and denote all E-F-extensional relations set by $F(X \times Y, E, F, L)$.

Definition 2.16. [4] Let $(X, E)$ and $(Y, F)$ be two TL-equivalence relations and $f \in F(X \times Y, E, F, L)$ then

a) $\mathrm{f}$ is called partial TL-function if $T\left(f(x, y), f\left(x, y^{\prime}\right)\right) \leq F\left(y, y^{\prime}\right)$ is satisfied $\forall x \in$ $X, y, y^{\prime} \in Y$.

b) $\mathrm{f}$ is called fully defined if $\mathrm{f}$ fulfills the condition $\bigvee_{z \in Y} f(x, z)=1$ for all $x \in X$.

c) A fully defined partial TL-function is called a TL-function. 
Definition 2.17. [5] Let $f \in F(X \times Y, E, F, L)$ be TL-function

a) $\mathrm{f}$ is called surjective if and only if $\bigvee_{x \in X} f(x, y)=1$ for all $y \in Y$.

b) $\mathrm{f}$ is called injective if and only if $T\left(f(x, y), f\left(x^{\prime}, y\right)\right) \leq E\left(x, x^{\prime}\right)$ for all $x, x^{\prime} \in X$ and $y \in Y$.

Theorem 2.18. [14] Let $(X, E)$ and $(Y, F)$ be two $T L$-equaivalence relations $f \in$ $F(X \times Y, L)$ and $A \in F(X, L)$. If $T$ is an infinetly $\vee$-distributive $t$-norm and $A$ is $E$-extensional then $f(A)$ is an $F$-extensional $L$-subset.

Theorem 2.19. [14] Let $(X, E)$ and $(Y, F)$ be two TL-equaivalence relations $f \in$ $F(X \times Y, L)$ and $B \in F(Y, L)$. If $T$ is an infinitely $\vee$-distributive $t$-norm and $B$ is $F$-extensional then $f^{-1}(B)$ is a E-extensional L-subset.

Theorem 2.20. [14] Let $f \in F(X \times Y, E, F, L), g \in F(Y \times Z, E, G, L)$ be $T L$ functions and $T$ be an infinitely $\vee$-distributive $t$-norm then $g{ }^{\circ} T \in F(X \times$ $Z, E, G, L)$ is a TL-function.

Definition 2.21. [2] Let $\mathrm{F}$ is a field. Then a L-subset $\mu: E \rightarrow L$ of a vector space $\mathrm{E}$ is a TL-subspace of $\mathrm{E}$ if for any $a, b \in F$ and $x, y \in E$ the following condition holds:

$$
\mu(a x+b y) \geq T(\mu(x), \mu(y)) .
$$

Theorem 2.22. [2] Let $\mu$ be a TL-subspace of a vector space E. Then

i) $\mu(0)=\sup \{\mu(x) \mid x \in X\}$,

ii) $\mu(a x)=\mu(x)$ for all $a \in F \backslash\{0\}$ and $x \in E$,

iii) If $x, y \in E$ and $\mu(x) \neq \mu(y)$ then $\mu(x+y)=T(\mu(x), \mu(y))$.

\section{Fuzzy Linear Mappings}

In this section we give definiton of TL-linear mappings. In literature some researchers gave fuzzy linear mappings by using classic mappings [9]. In this study we give a different fuzzy linear mapping by using the definition of fuzzy functions of Demirci [5]. With that definitions we give some theorems in classical algebra about linear mappings that can be moved to fuzzy algabra and proof them.

Definition 3.1. Let $\mathrm{X}$ be an E-vector space and $\mathrm{Y}$ be a F-vector space. Then a TL-function $f: X \times Y \rightarrow L$ is a TL-linear mapping if for any $x, x^{\prime} \in X, y, y^{\prime} \in$ $Y, a \in E, b \in F$ the following conditions hold:

i) $f\left(x+x^{\prime}, y+y^{\prime}\right) \geq T\left(f(x, y), f\left(x^{\prime}, y^{\prime}\right)\right)$,

ii) $f(a x, b y) \geq f(x, y)$.

If $f: X \times Y \rightarrow[0,1]$ is a fuzzy function with the above conditions then $\mathrm{f}$ is called a fuzzy linear mapping.

Theorem 3.2. Let $f: X \times Y \rightarrow L$ be a TL-linear mapping. Then for all $x \in$ $X, y \in Y$

i) $f\left(0_{X}, 0_{Y}\right) \geq f(x, y)$,

ii) $f(-x,-y)=f(x, y)$.

Proof. i) $x \in X, y \in Y f\left(0_{X}, 0_{Y}\right)=f(0 . x, 0 . y) \geq f(x, y)$.

ii) $f(-x,-y)=f((-1) \cdot x,(-1) \cdot y) \geq f(x, y)$

$f(x, y)=f((-1) \cdot(-x),(-1) \cdot(-y)) \geq f(-x,-y)$. 
Theorem 3.3. Let $f: X \times Y \rightarrow L$ be a TL-linear mapping and $T$ be a infinetly $\vee$-distributive t-norm. If $A: X \rightarrow L$ is TL-subspace of $E$ and $B: Y \rightarrow L$ is $T L$-subspace of $F$ then $f(A) T L$-subset is TL-subspace of $F$ and $f^{-1}(B)$ TL-subset is TL-subspace of $E$.

Proof. For all $a, b \in F, y, y^{\prime} \in Y$

$$
\begin{aligned}
& f(A)\left(a y+b y^{\prime}\right)=\bigvee_{x \in X} T\left(A(x), f\left(x, a y+b y^{\prime}\right)\right) \\
&= \bigvee_{x, x^{\prime} \in X} T\left(A\left(x+x^{\prime}\right), f\left(x+x^{\prime}, a y+b y^{\prime}\right)\right) \geq \bigvee_{x, x^{\prime} \in X} T\left(T\left(A(x), A\left(x^{\prime}\right)\right), T\left(f(x, y), f\left(x^{\prime}, y^{\prime}\right)\right)\right) \\
&= \bigvee_{x, x^{\prime} \in X} T\left(T(A(x), f(x, y)), T\left(A\left(x^{\prime}\right), f\left(x^{\prime}, y^{\prime}\right)\right)\right) \\
&= T\left(\bigvee_{x \in X} T(A(x), f(x, y)), \bigvee_{x^{\prime} \in X} T\left(A\left(x^{\prime}\right), f\left(x^{\prime}, y^{\prime}\right)\right)\right) \\
&= T\left(f(A)(y), f(A)\left(y^{\prime}\right)\right) . \text { Then } f(A) \text { is TL-subspace of F. } \\
& \text { For all } a, b \in E, x, x^{\prime} \in X \\
& f^{-1}(B)\left(a x+b x^{\prime}\right)=\bigvee_{y \in Y} T\left(B(y), f\left(a x+b x^{\prime}, y\right)\right) \\
&=\bigvee_{y, y^{\prime} \in Y} T\left(B\left(y+y^{\prime}\right), f\left(a x+b x^{\prime}, y+y^{\prime}\right)\right) \geq \bigvee_{y, y^{\prime} \in Y} T\left(T\left(B(y), B\left(y^{\prime}\right), T\left(f(x, y), f\left(x^{\prime}, y^{\prime}\right)\right)\right)\right) \\
&=\bigvee_{y, y^{\prime} \in Y} T\left(T(B(y), f(x, y)), T\left(B\left(y^{\prime}\right), f\left(x^{\prime}, y^{\prime}\right)\right)\right) \\
&= T\left(\bigvee_{y \in Y} T(B(y), f(x, y)), \bigvee_{y^{\prime} \in Y} T\left(B\left(y^{\prime}, f\left(x^{\prime}, y^{\prime}\right)\right)\right)\right) \\
&= T\left(f^{-1}(B)(x), f^{-1}(B)\left(x^{\prime}\right)\right) . \text { Then } f^{-1}(B) \text { is TL-subspace of E. }
\end{aligned}
$$

Theorem 3.4. Let $f: X \times Y \rightarrow L$ anb $g: Y \times Z \rightarrow L$ be a TL-linear mapping and $T$ be a infinetly $\vee$-distributive t-norm. Then $g \circ_{T} f: X \times Z \rightarrow L$ is a TL-linear mapping.

Proof. For any $x, x^{\prime} \in X, z, z^{\prime} \in Z, a \in E, b \in F$,

i) $g \circ_{T}\left(x+x^{\prime}, z+z^{\prime}\right)=\bigvee_{y \in Y} T\left(f\left(x+x^{\prime}, y\right), g\left(y, z+z^{\prime}\right)\right)$

$$
\begin{aligned}
& =\bigvee_{y, y^{\prime} \in Y} T\left(f\left(x+x^{\prime}, y+y^{\prime}\right), g\left(y+y^{\prime}, z+z^{\prime}\right)\right) \\
& \geq \bigvee_{y, y^{\prime} \in Y} T\left(T\left(f(x, y), f\left(x^{\prime}, y^{\prime}\right)\right), T\left(g(y, z), g\left(y^{\prime}, z^{\prime}\right)\right)\right) \\
& =\bigvee_{y, y^{\prime} \in Y} T\left(T(f(x, y), g(y, z)), T\left(f\left(x^{\prime}, y^{\prime}\right)\right)\right) \\
& =T\left(\bigvee_{y \in Y} T(f(x, y), g(y, z)), \bigvee_{y^{\prime} \in Y} T\left(f\left(x^{\prime}, y^{\prime}\right), g\left(y^{\prime}, z^{\prime}\right)\right)\right)
\end{aligned}
$$

$T\left(\left(g \circ_{T} f\right)(x, z),\left(g \circ_{T} f\right)\left(x^{\prime}, z^{\prime}\right)\right)$.

ii) $\left(g \circ_{T} f\right)(a x, b z)=\bigvee_{y^{\prime} \in Y} T(f(a x, y), g(y, b z)) \geq \bigvee_{y^{\prime} \in Y} T(f(a x, a y), g(b y, b z))$

$\geq \underset{y^{\prime} \in Y}{\bigvee} T(f(x, y), g(y, z))=(g \circ T f)(x, z)$.

the conditions are proved. Then $\left(g \circ_{T} f\right)$ is a TL-linear mapping.

Definition 3.5. Let $f \in F(X \times Y, E, F, L)$ be a TL-linear mapping.

Define $\operatorname{Kerf}: X \rightarrow L$ and $\operatorname{Imf}: Y \rightarrow L$ as follows.

$(K e r f)(x)=f(x, 0)$ and 
$(\operatorname{Imf})(y)=\bigvee_{x \in X} f(x, y)$

where $x \in X$ and $y \in Y$. Kerf is called the kernel of $\mathrm{f}$ and Imf is called the image of $f$.

Theorem 3.6. Let $f \in F(X \times Y, E, F, L)$ be a TL-linear mapping. Then;

i) Kerf is a TL-supspace of $X$,

ii) Imf is a TL-supspace of $Y$.

Proof. i) For all $a, b \in E$ and $x, y \in X$,

$(K e r f)(a x+b y)=f(a x+b y, 0)=f(a x+b y, 0+0) \geq T(f(a x, 0), f(b y, 0))$

$\geq T(f(x, 0), f(y, 0))=T((\operatorname{Ker} f)(x),(\operatorname{Ker} f)(y))$.

ii) For all $a, b \in F$ and $x, y \in Y$,

$$
\begin{aligned}
& (\operatorname{Im} f)(a x+b y)=\bigvee_{z \in X} f(z, a x+b y) \geq \bigvee_{z, z^{\prime} \in X} f\left(a z+b z^{\prime}, a x+b y\right) \\
& \geq \bigvee_{z, z^{\prime} \in X} T\left(f(a z, a x), f\left(b z^{\prime}, b y\right)\right) \geq \bigvee_{z, z^{\prime} \in X} T\left(f(z, x), f\left(z^{\prime}, y\right)\right)=T((\operatorname{Im} f)(x),(\operatorname{Im} f)(y)) .
\end{aligned}
$$

\section{REFERENCES}

[1] Kim S. H., Monk B., Neggers J., On pseudo fuzzy linear mappings, Information Sciences, 177, 897-905, (2007).

[2] Katsaras A. K., Liu D.B., Fuzzy Vector Spaces and Fuzzy Topological Vector Spaces, Journal of Mathematical Analysis and Applications, 58, 135-146, (1977).

[3] Muganda G. C., Fuzzy Linear and Affine Spaces, Fuzzy Sets and Systems, 38, 365-373, (1990).

[4] Demirci M., Recasens J., Fuzzy Groups, Fuzzy Functions and Fuzzy Equivalence Relations, Fuzzy Sets and Systems, 144, 441-458,(2004).

[5] Demirci M., Fuzzy Functions and Their Applications, Journal of Mathematical Analysis and Applications, 252, 495-517, (2000).

[6] Demirci M., Fundamentals of M-vague Algebra and M-vague Arithmetic Operations, Int. J. Uncertainly Fuzziness Knowledge-Based Systems, 10, 1, 25-75, (2002).

[7] $\breve{S}$ ostak A. P., Fuzzy Functions and an Extension of the Category L-Top of Chang-Goguen L-Topological Spaces, Proceedings of the Ninth Prague Symposium, pp. 271-294, Topological Atlas, Toronto, (2002).

[8] L. A. Zadeh, Fuzzy sets, Information and Control, 8,338-353, (1965).

[9] Abdukhalikov, K. S., The Dual of Fuzzy Subspace, Fuzzy Sets and Systems, 82, 375-381, (1996).

[10] Klement E.P., Mesiar R., Pap E., Triangular Norms. Position Paper I: Basic Analytical and Algebraic Properties, Fuzzy Sets and Systems, 145, 411-438, (2004).

[11] Klement E.P., Mesiar R., Pap E., Triangular Norms. Position Paper II:General Contructions and Parameterize Families, Fuzzy Sets and Systems, 145, 411-438, (2004).

[12] Klement E.P., Mesiar R., Pap E., Triangular Norms. Position Paper III: Continuous t-norms, Fuzzy Sets and Systems, 145, 439-454, (2004).

[13] F. Karaçal, D. Khadjiev, $\vee$-Distributive and infinitely $\vee$-distributive t-norms on complete lattice, Fuzzy Sets and Systems, 151, 341-352, (2005).

[14] Ü. Deniz, Different Approximation to Fuzzy Ring Homomorphisms, Sakarya University Journal of Science, 6,1163-1172,(2019).

[15] Rosenfeld A., Fuzzy groups, J. Math. Anal. Appl. 35, 512-517, (1971).

[16] Liu W.J., Fuzzy Invariant supgroups and fuzzy ideals, Fuzzy Sets and Systems 8, 133-139, (1982).

[17] Liu W.J., Operations on fuzzy ideals, Fuzzy Sets and Systems 11, 31-41, (1983).

Recep Tayyip ErdoĞan University, Mathematics Department, 53100, Rize, Turkey

Email address: umit.deniz@erdogan.edu.tr 